\title{
Dois Surtos de Escabiose num Hospital Terciário em Portugal
}

\section{Two Scabies Outbreaks at a Tertiary Care Hospital in Portugal}

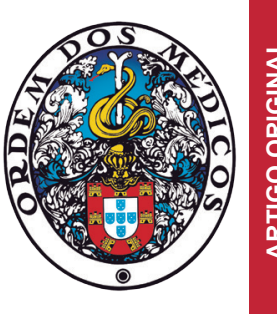

\author{
Nuno GOMES $\varangle^{1}$, Pedro Moura GUEDES ${ }^{2}$, Pedro NORTON ${ }^{2}$, Filomena AZEVEDO ${ }^{1}$, Carmen LISBOA ${ }^{1,3}$ \\ Acta Med Port 2020 Dec;33(12):803-810 - https://doi.org/10.20344/amp.12840
}

\begin{abstract}
RESUMO
Introdução: Os surtos de escabiose em instituições são um problema emergente. Determinar a melhor estratégia para o seu controlo é uma questão atual. Analisámos dois surtos hospitalares de escabiose e revimos a abordagem de surtos institucionais de escabiose. Material e Métodos: Realizámos um estudo observacional retrospetivo de dois surtos independentes de escabiose que ocorreram num hospital terciário português em 2018. Após a identificação dos casos-índice, dos casos de escabiose e dos indivíduos expostos, calculámos a taxa de ataque em doentes e em profissionais de saúde. Avaliámos ainda o tratamento e medidas de controlo de infeção, bem como o custo global de cada surto.

Resultados: Os surtos de escabiose ocorreram em duas enfermarias de Medicina Interna. Ambos tiveram como caso-índice doentes institucionalizados e com dermatose no momento do internamento. Na enfermaria 1 foram identificados 409 indivíduos expostos, 14 casos de escabiose e a taxa de ataque foi 3,4\%. Na enfermaria 2 foram identificados 254 indivíduos expostos, 17 casos e a taxa de ataque foi $6,7 \%$. Foi realizado o tratamento tópico dos casos e foram implementados cuidados ambientais.

Discussão: Na nossa análise, ambos os surtos tiveram como caso-índice doentes previamente institucionalizados e tiveram um impacto significativo, com centenas de indivíduos expostos e custos consideráveis. A análise de surtos hospitalares de escabiose é fundamentalmente retrospetiva e representa uma oportunidade para rever a estratégia da sua abordagem.

Conclusão: É premente a implementação de linhas de orientação sobre a abordagem de surtos institucionais de escabiose.

Palavras-chave: Escabiose; Portugal; Surtos de Doença
\end{abstract}

\section{ABSTRACT}

Introduction: Scabies outbreaks in healthcare institutions are an emerging problem. To determine the best management strategy is a topical matter. We analyzed two hospital scabies outbreaks and reviewed the management strategy of institutional scabies outbreaks. Material and Methods: We performed an observational retrospective study of two independent scabies outbreaks that occurred in a Portuguese tertiary hospital in 2018. Following the identification of the index cases, scabies cases and exposed individuals, we calculated the attack rate in patients and professionals. We also evaluated the treatment and infection control measures, as well as the global cost of each outbreak.

Results: The hospital outbreaks of scabies occurred in two wards of Internal Medicine. Both had as index cases institutionalized patients with dermatosis at the time of admission. In the Ward 1, there have been identified 409 exposed individuals, 14 cases of scabies and the attack rate was $3.4 \%$. In the Ward 2, there have been identified 254 exposed individuals, 17 cases and the attack rate was $6.7 \%$. Topical treatment was prescribed to the cases and environmental measures were implemented.

Discussion: In our analysis, both outbreaks had as index cases institutionalized patients and had a significant impact, with hundreds of exposed individuals and considerable costs. The analysis of hospital scabies outbreaks is mostly retrospective and represents an opportunity to review its best management strategy.

Conclusion: Implementation of guidelines on tackling scabies outbreaks in institutional settings is urgent.

Keywords: Disease Outbreaks; Portugal; Scabies

\section{INTRODUÇÃO}

A escabiose é uma infeção parasitária comum causada pelo ácaro Sarcoptes scabiei variante hominis. Afeta ambos os géneros e ocorre em todas as idades, grupos étnicos e níveis socioeconómicos. Estima-se uma prevalência global de 300 milhões de casos por ano. ${ }^{1} \mathrm{Na}$ Europa Ocidental, a prevalência estimada é inferior a $1 \%{ }^{2}$ O diagnóstico é clínico e carateriza-se pela tríade de pápulas inflamatórias pruriginosas que surgem principalmente nas pregas interdigitais, punhos, axilas, mamilos e região anogenital nos adultos com contexto epidemiológico positivo. No entanto, pode ter apresentações atípicas nos doentes idosos e adultos imunocomprometidos. ${ }^{1,2} \mathrm{O}$ exame microscópico do raspado das lesões e a dermatoscopia são aconselhados para confirmar o diagnóstico clínico. ${ }^{1,3}$

$\mathrm{Na}$ escabiose clássica, cerca de cinco a 15 ácaros fêmeas habitam no hospedeiro infetado; porém, esse número pode atingir centenas ou milhares de ácaros na escabiose crostosa, uma forma extremamente contagiosa. O período de incubação varia entre três e seis semanas após a infeção primária, podendo a transmissão ser direta (contacto pele-a-pele) ou indireta (roupas ou objetos contaminados). Sendo o diagnóstico clínico, é frequente ocorrerem atrasos e erros diagnósticos. Para um tratamento eficaz, quer os fármacos tópicos e/ou orais, quer as medidas de prevenção

1. Serviço de Dermatovenereologia. Centro Hospitalar Universitário de São João. Porto. Portugal.

2. Serviço de Saúde Ocupacional. Centro Hospitalar Universitário de São João. Porto. Portugal.

3. Divisão de Microbiologia. Departamento de Patologia e Centro de Investigação em Tecnologias e Serviços de Saúde. Faculdade de Medicina. Universidade do Porto. Porto. Portugal.

$\triangle$ Autor correspondente: Nuno Gomes. nunompretogomes@gmail.com

Recebido: 16 de setembro de 2019 - Aceite: 04 de dezembro de 2019 | Copyright $\odot$ Ordem dos Médicos 2020 
ambientais, são essenciais. ${ }^{1}$

Os surtos de escabiose são um problema emergente nas sociedades desenvolvidas. ${ }^{4}$ Porém, estes surtos são muitas vezes subestimados e são reconhecidos muito tardiamente. $\mathrm{O}$ risco de surtos é particularmente alto em instituições como hospitais, lares, prisões e locais de alojamento turístico. Neste contexto, os surtos podem ser extensos devido às caraterísticas do parasita e ao curso clínico da doença. Fatores como o longo período de incubação, a clínica inespecífica, a ausência de um marcador biológico, a reinfestação frequente associada a período de incubação mais curto e/ou as complicações dos tratamentos tópicos que simulam reinfestações, são fatores que favorecem a ocorrência de surtos.

A abordagem dos surtos institucionais de escabiose não está bem definida, sendo essencial determinar qual a melhor estratégia a adotar e homogeneizar os protocolos institucionais. Na última década, a emergência de surtos institucionais implicou a revisão sobre o que se sabe sobre escabiose e a consciencialização das múltiplas questões não resolvidas acerca da melhor abordagem destes surtos. Alguns autores defendem a profilaxia em massa de todos os expostos ${ }^{2}$; outros, advogam uma estratégia baseada na informação em massa. ${ }^{5} \mathrm{O}$ nosso objetivo foi realizar um estudo observacional retrospetivo de dois surtos independentes de escabiose que ocorreram num hospital terciário português em 2018. Como objetivo secundário propusemo-nos a rever a literatura e propor uma abordagem de surtos institucionais de escabiose em Portugal.

\section{MATERIAL E MÉTODOS}

Realizámos um estudo observacional retrospetivo de dois surtos de escabiose que ocorreram em abril de 2018 em duas enfermarias de Medicina Interna (Enfermaria 1 e Enfermaria 2) do Centro Hospitalar Universitário São João (CHUSJ), Porto.

Procedemos à identificação precisa dos casos-índice (Cl) com base na revisão dos processos clínicos e reuniões com as equipas médica e de enfermagem de ambas enfermarias. Identificámos todos os profissionais expostos e todos os doentes internados na mesma enfermaria dos $\mathrm{Cl}$ por revisão dos processos clínicos dos doentes, das escalas de trabalho e processos clínicos dos profissionais.

Os parâmetros que avaliámos incluíram a duração do surto, o número de doentes e profissionais expostos, o número de casos de escabiose, o tratamento e medidas de controlo de infeção preconizados, bem como a abordagem dos conviventes.

Dado o período de incubação do agente etiológico, ${ }^{1}$ o tempo de análise incluiu as seis semanas anteriores à data do diagnóstico dos $\mathrm{Cl}$ e prolongou-se até às seis semanas após o último caso diagnosticado, altura em que os surtos foram declarados como resolvidos..$^{6,7}$

Efetuámos também uma análise do custo global dos surtos de escabiose que incluiu o custo do tratamento, do absentismo dos profissionais, das consultas médicas e das medidas ambientais de isolamento de contacto. Para ana- lisar o custo dos tratamentos para a escabiose disponíveis em Portugal, fizemos uma pesquisa em várias farmácias comunitárias da região do Grande Porto.

Por último, visitámos as instituições de residência/de origem dos $\mathrm{Cl}$ (um $\mathrm{Cl}$ estava institucionalizado num hospital psiquiátrico e outro $\mathrm{Cl}$ num lar de terceira idade).

\section{Definições}

Por 'casos' de escabiose, designámos os doentes ou profissionais com escabiose no contexto do surto e cuja doença foi confirmada por médico dermatologista (com apoio de raspado/dermatoscopia, se necessário).

Por 'expostos' à escabiose, designámos os doentes internados na mesma enfermaria dos $\mathrm{Cl}$ ou os profissionais que lá trabalharam durante o surto. Os doentes internados no mesmo quarto dos $\mathrm{Cl}$ ou os profissionais que lhes prestaram assistência direta foram designados por 'expostos diretos'.

A 'taxa de ataque' para os profissionais e doentes foi calculada dividindo o número de casos de escabiose pelo número total de profissionais ou doentes expostos.

Por 'profissionais', designámos todos os profissionais de saúde, incluindo os médicos, enfermeiros, assistentes operacionais e pessoal de limpeza, envolvidos epidemiologicamente no surto.

Este estudo foi aprovado pela Comissão de Ética para a Saúde do Centro Hospitalar de São João/ Faculdade de Medicina da Universidade do Porto. Todos os procedimentos cumpriram os princípios éticos e foi assegurada a confidencialidade dos dados no contexto de uma análise retrospetiva.

\section{RESULTADOS}

Em abril de 2018, ocorreram dois surtos independentes de escabiose em duas enfermarias de Medicina Interna do CHUSJ, Enfermarias 1 e 2, situadas em pisos diferentes. Estas enfermarias têm, respetivamente, 116 e 100 camas, e equipas de profissionais independentes.

$\mathrm{O} \mathrm{Cl}$ do surto da Enfermaria 1 foi um homem de 32 anos que esteve internado entre os dias 2 e 23 de abril por infeção respiratória. O doente tinha, como antecedentes, debilidade intelectual e epilepsia (secundárias a meningite na infância), estando institucionalizado num hospital psiquiátrico desde há vários anos, local onde se registaram surtos de escabiose no início de 2018. À data de entrada na Enfermaria, o doente tinha dermatose pruriginosa, mas o diagnóstico de escabiose foi realizado cerca de 15 dias depois (Fig. 1). Foram identificados 409 indivíduos expostos, 243 doentes e 166 profissionais, dos quais 14 foram casos de escabiose. Todos os casos ocorreram em profissionais, 13 dos quais enfermeiros. A taxa de ataque global nos profissionais foi de $8,4 \%$ (Tabela 1).

$\mathrm{O} \mathrm{Cl}$ do surto da Enfermaria 2 foi uma mulher de 83 anos que esteve internada na Enfermaria 2 entre os dias 19 e 24 de abril por acidente vascular cerebral hemorrágico. A doente tinha como antecedentes, síndrome demencial e diabetes mellitus tipo 2, estando institucionalizada num lar. 


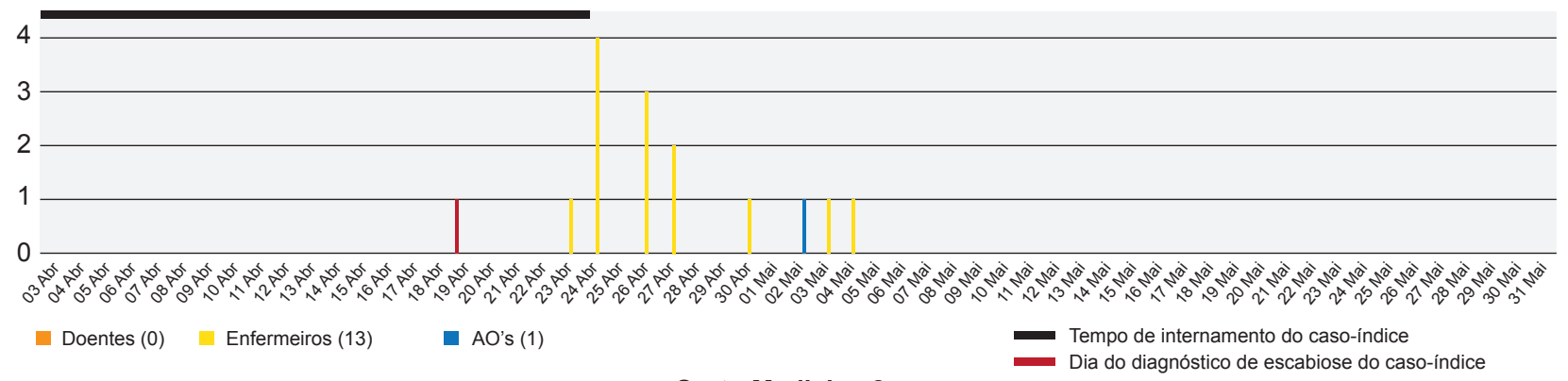

Surto Medicina 2

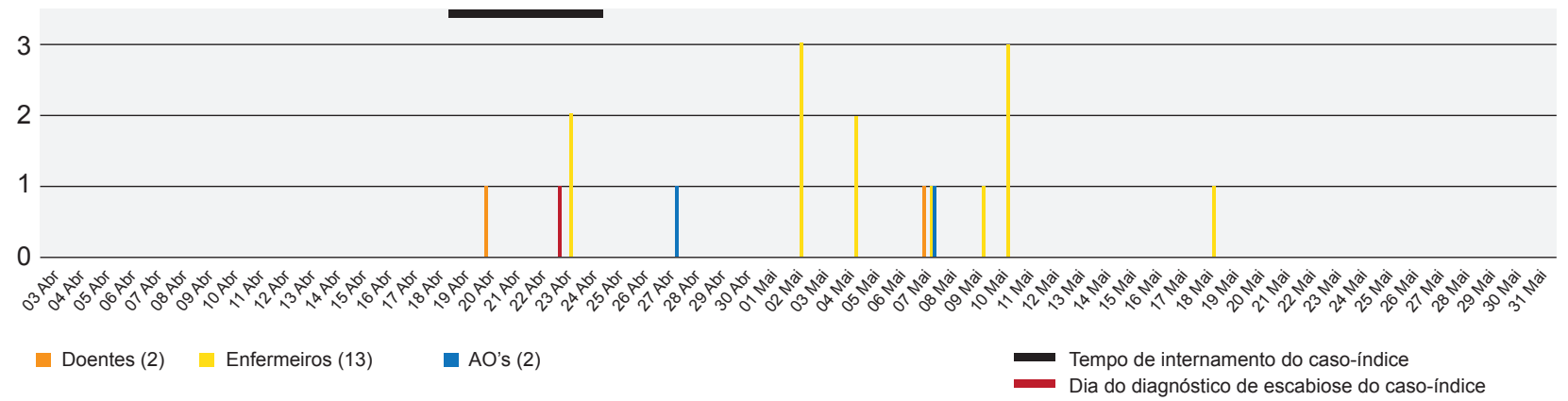

Figura 1 - Evolução temporal dos 2 surtos de escabiose ocorridos no CHUSJ em 2018

$\mathrm{AO}$ : assistentes operacionais

Um mês antes do internamento havia sido diagnosticada com escabiose no Serviço de Urgência do CHUSJ. À entrada na Enfermaria a dermatose foi identificada tendo o diagnóstico de escabiose sido realizado após quatro dias de internamento (Fig. 1). Foram identificados 254 indivíduos expostos, 106 doentes e 148 profissionais, dos quais 17 foram casos de escabiose: dois em doentes e 15 em profissionais, 13 dos quais enfermeiros. A taxa de ataque global nos profissionais foi de $10,1 \%$ e nos doentes foi de 1,9\% (Tabela 1).

Os casos-índice e os dois casos de escabiose em doentes foram tratados com enxofre tópico a $8 \%$ três dias consecutivos, com repetição do tratamento na semana seguinte. Nenhum destes doentes teve novos sintomas/sinais de escabiose após o tratamento efetuado. Os doentes foram mantidos em isolamento de contacto pelo menos 24 horas após a primeira aplicação do tratamento. As medidas imediatas de controlo de infeção incluíram a lavagem da roupa de cama e vestuário dos casos de escabiose à temperatura de $60^{\circ} \mathrm{C}$. Em nenhum dos surtos houve necessidade de encerrar quartos das enfermarias ou de protelar a alta clínica.

Os 29 profissionais com escabiose foram observados no Serviço de Saúde Ocupacional e medicados topicamente com enxofre de $6 \%$ a $10 \%$ (26 profissionais) ou benzoato de benzilo (três profissionais). Foi também prescrito o mesmo tratamento a todos os seus conviventes diretos. Os profissionais foram informados sobre os cuidados ambientais a adotar. Foi declarada uma incapacidade temporária absoluta a cada um dos profissionais, com um período de 24 horas após o início da aplicação do tratamento.

A estratégia da equipa de abordagem do surto, constituída por médicos de Dermatovenereologia e Saúde Ocupacional, baseou-se na informação dirigida aos profissionais e doentes das enfermarias envolvidas. Apenas foi preconizado o tratamento dos casos de escabiose e seus conviventes.

Em relação à análise do custo do surto, avaliámos os custos da abstenção laboral diária dos profissionais

Tabela 1 - Número de expostos, expostos diretos, casos e taxas de ataque dos dois surtos de escabiose ocorridos no CHUSJ em 2018

\begin{tabular}{|c|c|c|c|c|}
\hline Surto & Indivíduos & Doentes & Profissionais & Total \\
\hline \multirow{4}{*}{ Enfermaria 1} & Expostos & 243 & 166 & 409 \\
\hline & Expostos diretos & 8 & 38 & 46 \\
\hline & Casos & 0 & 14 & 14 \\
\hline & Taxa de ataque & $0 \%$ & $8,4 \%$ & $3,4 \%$ \\
\hline \multirow{4}{*}{ Enfermaria 2} & Expostos & 106 & 148 & 254 \\
\hline & Expostos diretos & 7 & 26 & 33 \\
\hline & Casos & 2 & 15 & 17 \\
\hline & Taxa de ataque & $1,9 \%$ & $10,1 \%$ & $6,7 \%$ \\
\hline
\end{tabular}


afetados (€1100), do tratamento tópico dos casos de escabiose com enxofre ou benzoato de benzilo (€1100) e das consultas médicas efetuadas a profissionais e a doentes (€350). A estimativa do custo global de ambos os surtos, tendo em conta as variáveis mencionadas, cifrou-se em cerca de $€ 2350$ (Tabela 2).

$\mathrm{Na}$ análise do custo dos tratamentos para a escabiose disponíveis em Portugal, constatámos que a ivermectina oral (manipulada na farmácia da comunidade) e o benzoato de benzilo tópico foram os fármacos com preço mais reduzido (Tabela 3).

Na Tabela 4 estão resumidas as características dos surtos de escabiose publicados mais recentemente. .,7-14 $^{-1}$

Nas visitas às instituições de origem dos $\mathrm{Cl}$, foi constatado que havia história de escabiose em vários institucionalizados, com tratamento tópico (benzoato de benzilo) efetuado em tempos diferentes. Verificámos as dificuldades em cumprir as medidas ambientais de evicção e rastreio de conviventes e visitas.

\section{DISCUSSÃO}

Os dois surtos hospitalares que analisámos têm algumas caraterísticas que os diferenciam de outros surtos reportados. A duração dos nossos surtos foi bastante inferior à da maioria dos surtos publicados, o que se pode explicar pelo aumento da suspeição diagnóstica e do alarmismo dos profissionais após o diagnóstico do $\mathrm{Cl}$ da Enfermaria 1 , tendo permitido a identificação precoce do surto da Enfermaria 2. Por outro lado, os $\mathrm{Cl} 1$ e 2, apesar de provavelmente terem escabiose desde há alguns meses antes do internamento, não foram diagnosticados com escabiose crostosa e consequentemente apresentavam menor carga infeciosa. Pelo contrário, dos nove surtos publicados, seis deles correspondiam a $\mathrm{Cl}$ com escabiose crostosa.

O nosso estudo foi observacional. Registámos taxas de ataque superiores para os profissionais relativamente aos doentes, o que está de acordo com o descrito e se explica sobretudo pela rotatividade dos profissionais por vários doentes da mesma enfermaria. A nossa abordagem aos surtos centrou-se na informação aos doentes e profissionais e tratamento dos casos de escabiose confirmados por dermatologista, bem como prescrição de tratamento aos seus conviventes diretos. Porém, muitos autores defendem a profilaxia em massa de todos os contactos.

As taxas de ataque globais dos nossos surtos $(3,4 \%$ e $6,7 \%$ ) são também inferiores à da maioria dos surtos publicados. Numa revisão sobre 84 surtos institucionais de escabiose, 33 dos quais hospitalares ${ }^{6}$ os surtos persistiram por três meses e a taxa de ataque média foi de $38 \%$; o $\mathrm{Cl}$ tinha escabiose crostosa em $83 \%$ dos casos. ${ }^{6}$ Como referimos, o facto dos nossos $\mathrm{Cl}$ terem menor carga infeciosa em relação a indivíduos com escabiose crostosa pode ter contribuído para o seu eficaz controlo mesmo sem profilaxia em massa. Acresce que ambos os $\mathrm{Cl}$ já tinham realizado tratamento anti-escabiótico previamente nas respetivas instituições. As medidas de isolamento de contacto e de informação aos doentes, profissionais e conviventes foram, certamente, essenciais. Apesar de uma revisão Cochrane ${ }^{15}$ de 2014 não ter comprovado que a profilaxia dos contactos é uma medida eficaz para prevenir a disseminação da escabiose, entendemos que essa medida também foi fulcral para o controlo do surto. O tratamento instituído na maioria dos casos foi o enxofre tópico, sobretudo pela sua eficácia e experiência acumulada. ${ }^{3}$ Porém, são reconhecidas as limitações logísticas dos tratamentos tópicos para a escabiose e a dificuldade do tratamento síncrono de todos os

Tabela 2 - Custos dos dois surtos de escabiose nos doentes e profissionais

\begin{tabular}{llll}
\hline Variáveis & Profissionais & Doentes & Custo \\
\hline Abstenção laboral diária & $26 \times € 40$ (enfermeiros) & & $€ 1100$ \\
& $3 \times € 20$ (assistentes operacionais) & & $€ 1087$ \\
Manipulado de enxofre & $€ 18,13 / 100 \mathrm{~g}$ & $€ 18,13 / 100 \mathrm{~g}$ & $€ 13$ \\
& Logo, $18,13 \times 2 \times 26$ & Logo, $18,13 \times 2 \times 4$ & $€ 350$ \\
Benzoato de benzilo & $€ 4,45 / 200 \mathrm{~mL}$ & - & $€ 2348$ \\
Consultas médicas & $€ 203$ & $€ 147$ & $€ 292$ \\
Custo total & $€ 2056$ & & \\
\hline
\end{tabular}

Tabela 3 - Estimativa do custo dos tratamentos anti-escabióticos tópicos e orais em Portugal

\begin{tabular}{|c|c|c|c|c|c|}
\hline & $\begin{array}{c}\text { Enxofre } 8 \% \\
\text { tópico }\end{array}$ & $\begin{array}{c}\text { Permetrina } 5 \% \\
\text { tópico }\end{array}$ & $\begin{array}{c}\text { Ivermectina } 1 \% \\
\text { tópico }\end{array}$ & $\begin{array}{l}\text { Benzoato de } \\
\text { benzilo tópico }\end{array}$ & $\begin{array}{l}\text { Ivermectina } \\
\text { oral }\end{array}$ \\
\hline $\begin{array}{l}\text { Preço parcial } \\
\text { (arredondado às } \\
\text { centésimas) do } \\
\text { produto }\end{array}$ & $\begin{array}{l}€ 25,02 \\
(100 \mathrm{~g})\end{array}$ & $\begin{array}{l}€ 26,61 \\
(100 \mathrm{~g})\end{array}$ & $\begin{array}{l}€ 80,92 \\
(100 \mathrm{~g})\end{array}$ & $\begin{array}{c}€ 8,98 \\
(200 \mathrm{~mL})\end{array}$ & $\begin{array}{c}€ 9,04 \\
(14 \mathrm{mg}-200 \mathrm{ug} \mathrm{kg} \\
\text { para doente de } 70 \mathrm{~kg})\end{array}$ \\
\hline $\begin{array}{l}\text { Preço total } \\
\text { (arredondado às } \\
\text { unidades) de um } \\
\text { tratamento }\end{array}$ & $€ 50,00$ & $€ 53,00$ & $€ 162,00$ & $€ 18,00$ & $€ 18,00$ \\
\hline
\end{tabular}




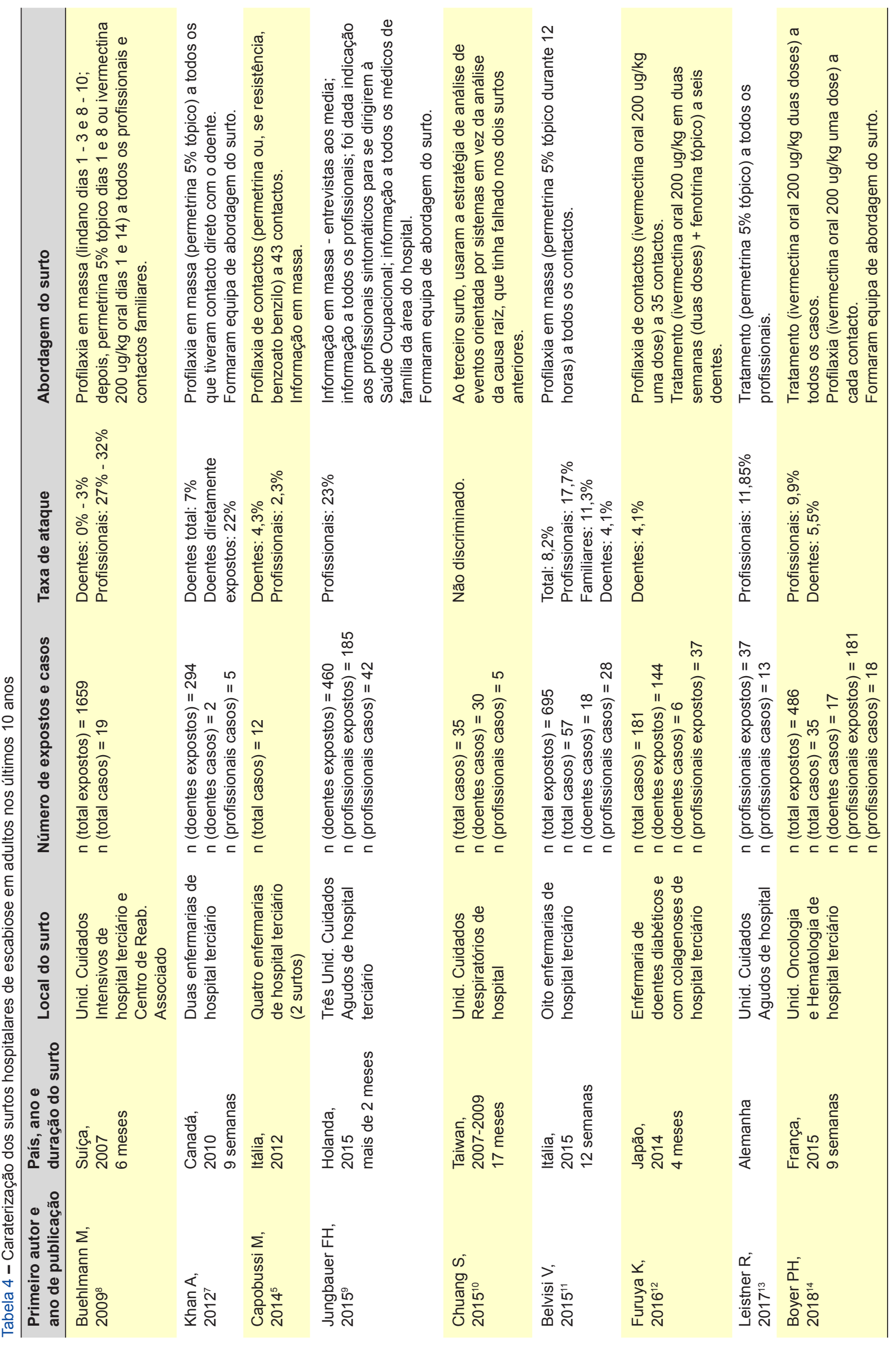


casos, as quais pudemos constatar in loco, quer na instituição psiquiátrica quer no lar que visitámos.

Em qualquer tipo de surto, a dificuldade de diagnóstico dos $\mathrm{Cl}$ é um fator a ter em conta. ${ }^{16,17}$ Frequentemente, e tal como ocorreu nos nossos $\mathrm{Cl}$, estes doentes têm síndrome demencial ou debilidade mental, sendo menos capazes de expressar os próprios sintomas. ${ }^{17}$

Dos nove artigos ${ }^{5,7-14}$ incluídos na revisão, nenhum versa sobre a realidade portuguesa, mas seis deles são de países europeus. As análises dos surtos são maioritariamente retrospetivas, com viés de seleção. Nem todas analisam apenas surtos hospitalares, pois envolvem frequentemente as unidades de reabilitação associadas. As definições aplicadas para 'expostos' e 'casos' são heterogéneas, assim como os critérios de diagnóstico da escabiose, o que dificulta a sua comparação. Para além disso, os próprios desenhos das análises são heterogéneos: algumas foram puramente observacionais, enquanto outras envolveram alguma intervenção ou questionários aos indivíduos expostos. Dos surtos publicados os custos foram atribuídos sobretudo ao absentismo dos profissionais, consultas médicas e tratamentos.

Em relação ao tratamento da escabiose, uma revisão Cochrane recente ${ }^{18}$ salientou que a ivermectina oral seria privilegiada em caso de grandes grupos de doentes ou perante dificuldade no cumprimento das instruções da aplicação tópica dos anti-escabióticos. Em Portugal, pela ausência de comercialização da ivermectina oral em humanos e da permetrina tópica a $5 \%$, o tratamento mais usado é o benzoato de benzilo; o enxofre é também muito usado em manipulado pela sua eficácia e segurança. ${ }^{4}$.

A abordagem específica dos surtos de escabiose tem ganho crescente importância nas linhas de orientação de tratamento. A Academia Europeia de Dermatovenereologia preconiza o tratamento em massa de grandes populações com doença endémica com dose única de ivermectina oral e defende que todos os indivíduos expostos devem ser tratados independentemente de apresentarem sintomas. ${ }^{3}$ Na Alemanha, ${ }^{2,19}$ país onde a escabiose não é doença de declaração obrigatória (tal como em Portugal), um surto de escabiose é indicação para substituição do tratamento com permetrina tópica por ivermectina oral, sobretudo por razões logísticas. Em outros países europeus, como a Itália, a escabiose é uma doença de declaração obrigatória. ${ }^{4,5}$ Neste caso, uma estratégia baseada na informação em massa pode ser eficaz. A disponibilidade dos tratamentos anti-escabióticos é também variável nos países europeus, o que torna mais difícil a aplicação nacional de linhas de orientação estrangeiras. Porém, alguns factos são unânimes, como o tratamento simultâneo dos conviventes expostos ou a premissa de que o prurido pós-escabiótico não é indicação para retratamento. , $^{3,21}$

Algumas publicações versam especificamente sobre o tratamento a aplicar em surtos de escabiose. White LC et $a^{22}$ reviram a abordagem dos surtos de escabiose em lares ingleses e concluíram que a maioria das linhas de orientação não avaliava adequadamente os desafios logísticos associados ao tratamento em massa. ${ }^{22,23}$ Por outro lado, as medidas ambientais são essenciais no controlo do surto, especialmente em contexto hospitalar. ${ }^{24}$ Salientamos que, ao contrário do que acontece em lares, ${ }^{25}$ instituições psiquiátricas, ${ }^{26}$ infantários ${ }^{27}$ ou contexto familiar, ${ }^{28}$ os hospitais têm uma população de doentes em mutação constante, o que torna a abordagem dos surtos muito mais complexa.

A avaliação dos surtos de escabiose nos países em desenvolvimento é também de grande importância. De facto, a escabiose é considerada uma dermatose tropical negligenciada, sendo um problema de saúde global. ${ }^{29,30}$ Como tal, os equipamentos diagnósticos a custo reduzido podem ser essenciais na abordagem dos surtos neste contexto..$^{31-34}$ Por outro lado, a administração de ivermectina oral em massa parece ser eficaz e é largamente adoptada. ${ }^{29,35} \mathrm{Po}-$ rém, o tratamento em massa pode induzir resistência aos tratamentos anti-escabióticos, que deve ser monitorizada. ${ }^{29}$ Nos Estados Unidos da América, estão planeados estudos clínicos com a moxidectina oral, com semi-vida mais longa que a ivermectina, que se pode revelar uma opção atrativa na profilaxia em grande escala. ${ }^{29}$

A nossa análise tem várias limitações, uma das quais é o seu desenho retrospetivo. É também discutível a perspetiva simplista de considerar como profissionais expostos apenas os médicos, enfermeiros, assistentes operacionais e pessoal de limpeza. Tendo em conta o modo de transmissão da escabiose, outros profissionais como pessoal da lavandaria, radiologistas ou outros técnicos que contactaram com os doentes e/ou suas roupas podem ter sido expostos. Ademais, também não foram considerados os indivíduos que visitaram os doentes internados, também eles possíveis veículos transmissores da escabiose. Por último, na análise de custos, a aplicação de emoliente ou dermocorticóide nos casos tratados com benzoato de benzilo (habitualmente associado a reações de eczematização) ${ }^{2}$ não foi incluída. Acresce também o custo das medidas ambientais de isolamento de contacto, bem como o tratamento prescrito para os conviventes, cujo custo extra não é menosprezável. ${ }^{36}$ Por último, não foi possível analisar todos os profissionais que recorreram às consultas de Dermatovenereologia e Saúde Ocupacional e que não foram diagnosticados com escabiose, cuja consulta se deveu ao alarme geral provocado pelos surtos. Este facto, aliado aos indivíduos que foram tratados para o prurido pós-escabiótico certamente contribuiu para o aumento do custo dos surtos.

Após o reconhecimento de um surto de escabiose, deve-se proceder urgentemente à constituição de uma equipa multidisciplinar, com médicos especialistas de Dermatovenereologia, Doenças Infecciosas, Saúde Ocupacional e Saúde Pública, bem como elementos da equipa de Enfermagem e do Conselho de Administração. Este é o primeiro passo para a eficaz abordagem do surto. ${ }^{2}$ A nosso ver, a profilaxia em massa de todos os expostos apenas deve ser equacionada se o surto tiver grande dimensão ou se o $\mathrm{Cl}$ tiver o diagnóstico de escabiose crostosa. Nesse caso, pelos resultados supra-invocados, nomeadamente o preço e facilidade logística, a ivermectina oral parece-nos uma 
opção melhor em relação à permetrina tópica. Caso não se adopte a profilaxia em massa, deve-se privilegiar uma estratégia baseada na informação em massa, medidas ambientais de evicção da exposição e tratamento apenas dos casos confirmados ou altamente suspeitos.

Ainda há múltiplas questões a serem clarificadas em linhas de orientação futuras da escabiose, nomeadamente o papel do dermatologista no diagnóstico e tratamento, a meIhor profilaxia a aplicar e o público-alvo da mesma, a própria definição dos surtos de escabiose, o período de follow-up necessário para declarar o fim do surto e a atribuição da responsabilidade dos custos financeiros do mesmo. ${ }^{15,22}$ Por último, os surtos de escabiose têm tendência a aumentar num futuro próximo como resultado do envelhecimento da população e aumento da densidade populacional nas áreas urbanas. ${ }^{37}$ Como tal, é essencial uma ação coordenada e concertada para controlar a escabiose a nível global. ${ }^{38}$ No futuro, a revisão dos surtos de escabiose, tão impactantes para doentes e profissionais, deve resultar em linhas de orientação específicas de abordagem do problema, que possam também ser aplicadas em outros contextos, como lares ou instituições psiquiátricas, e permitam evitar os fatores de risco para a falência do tratamento. ${ }^{39}$

\section{CONCLUSÃO}

São revistos dois surtos de escabiose independentes que ocorreram recentemente num hospital terciário português. Ambos os surtos tiveram um impacto significativo, com centenas de indivíduos expostos e custos consideráveis. As linhas de orientação internacionais são ainda vagas quanto à abordagem dos surtos de escabiose em contexto hospitalar. As revisões deste tipo de surtos e respetiva abordagem poderão eventualmente ser extrapoladas para instituições psiquiátricas, lares, prisões, entre outros. Novos estudos poderão aumentar o nosso conhecimento acerca da melhor abordagem de um problema tão emergente e recorrente nas instituições hospitalares. Urge a elaboração de linhas de orientação sobre a abordagem de surtos de escabiose em contextos institucionais.

\section{AGRADECIMENTOS}

Os autores gostariam de expressar o seu agradecimento a toda a equipa médica e de enfermagem das Enfermarias 1 e 2, cujo apoio foi essencial no desenvolver deste trabalho, bem como aos profissionais de saúde da instituição psiquiátrica e do lar dos casos-índice.

\section{PROTEÇÃO DE PESSOAS E ANIMAIS}

Os autores declaram que os procedimentos seguidos estavam de acordo com os regulamentos estabelecidos pelos responsáveis da Comissão de Investigação Clínica e Ética e de acordo com a Declaração de Helsínquia da Associação Médica Mundial actualizada em 2013.

\section{CONFIDENCIALIDADE DOS DADOS}

Os autores declaram ter seguido os protocolos do seu centro de trabalho acerca da publicação de dados.

\section{CONFLITO DE INTERESSES}

Os autores declaram a inexistência de conflitos de interesse na realização do presente trabalho.

\section{FONTES DE FINANCIAMENTO}

Não existiram fontes externas de financiamento para a realização deste artigo.

\section{REFERÊNCIAS}

1. Chosidow O. Scabies. N Engl J Med. 2006;354:1718-27

2. Stoevesandt J, Carlé L, Leverkus M, Hamm H. Control of large institutional scabies outbreaks: institutional scabies outbreaks. J Dtsch Dermatol Ges. 2012;10:637-47.

3. Salavastru CM, Chosidow O, Boffa MJ, Janier M, Tiplica GS. European guideline for the management of scabies. J Eur Acad Dermatol Venereol. 2017;31:1248-53.

4. Santiago $F$, Januário $G$. Escabiose: revisão e foco na realidade portuguesa. J Port Soc Dermatol Venereol. 2017;75:129-37.

5. Capobussi M, Sabatino G, Donadini A, Tersalvi CA, Castaldi S. Control of scabies outbreaks in an Italian hospital: an information-centered management strategy. Am J Infect Control. 2014;42:316-20.

6. Mounsey KE, Murray HC, King M, Oprescu F. Retrospective analysis of institutional scabies outbreaks from 1984 to 2013: lessons learned and moving forward. Epidemiol Infect. 2016;144:2462-71.

7. Khan A, O'Grady S, Muller MP. Rapid control of a scabies outbreak at a tertiary care hospital without ward closure. Am J Infect Control. 2012;40:451-5.

8. Buehlmann M, Beltraminelli $H$, Strub $C$, Bircher A, Jordan X, Battegay $M$, et al. Scabies outbreak in an intensive care unit with 1,659 exposed individuals - key factors for controlling the outbreak. Infect Control Hosp Epidemiol. 2009;30:354-60.

9. Jungbauer FH, Veenstra-Kyuchukova YK, Koeze J, KruijtSpanjer MR, Kardaun SH. Management of nosocomial scabies, an outbreak of occupational disease: management of nosocomial scabies. Am J Ind Med. 2015;58:577-82.

10. Chuang S, Howley PP, Lin SH. Implementing systems thinking for infection prevention: the cessation of repeated scabies outbreaks in a respiratory care ward. Am J Infect Control. 2015;43:499-505.

11. Belvisi V, Orsi GB, Del Borgo C, Fabietti P, lanari A, Albertoni F, et al. Large nosocomial outbreak associated with a norwegian scabies index case undergoing TNF- $\alpha$ inhibitor treatment: management and control. Infect Control Hosp Epidemiol. 2015;36:1358-60.

12. Furuya $\mathrm{K}$, Nakajima $\mathrm{H}$, Sasaki $\mathrm{Y}$, Ishiko A, Urita $\mathrm{Y}$. A scabies outbreak in a diabetic and collagen disease ward: management and prevention. Exp Ther Med. 2016;12:3711-5.

13. Leistner R, Buchwald D, Beyer M, Philipp S. Scabies outbreak among healthcare workers in a German acute care hospital. J Infect Prev. 2017;18:189-92.

14. Boyer PH, Deboscker S, Hernandez C, Ramsheyi M, Schneider P, Foeglé J, et al. An undiagnosed index case leading to a nosocomial scabies outbreak: how mass single-dose ivermectin treatment can help control a nosocomial epidemic. Infect Control Hosp Epidemiol. 2018;39:631-2.

15. FitzGerald D, Grainger RJ, Reid A. Interventions for preventing the spread of infestation in close contacts of people with scabies. Cochrane Database Syst Rev. 2014;2:CD009943.

16. Cassell JA, Middleton J, Nalabanda A, Lanza S, Head MG, Bostock J, et al. Scabies outbreaks in ten care homes for elderly people: a prospective study of clinical features, epidemiology, and treatment outcomes. Lancet Infect Dis. 2018;18:894-902.

17. Hewitt KA, Nalabanda A, Cassell JA. Scabies outbreaks in residential care homes: factors associated with late recognition, burden and impact. A mixed methods study in England. Epidemiol Infect. 2015;143:154251

18. Rosumeck S, Nast A, Dressler C. Ivermectin and permethrin for treating 
scabies. Cochrane Database Syst Rev. 2018;4:CD012994.

19. Sunderkötter C, Feldmeier H, Fölster-Holst R, Geisel B, KlinkeRehbein S, Nast A, et al. S1 guidelines on the diagnosis and treatment of scabies - short version: scabies guidelines. J Dtsch Dermatol Ges. 2016;14,1155-67.

20. Tavares M, Selores M. Escabiose - recomendações práticas para diagnóstico e tratamento. Nascer e Crescer. 2013;22:80-6.

21. Mara D. Scabies control: the forgotten role of personal hygiene. Lancet Infect Dis. 2018;18:1068.

22. White LC, Lanza S, Middleton J, Hewitt K, Freire-Moran L, Edge C, et al. The management of scabies outbreaks in residential care facilities for the elderly in England: a review of current health protection guidelines. Epidemiol Infect. 2016;144:3121-30.

23. Ladbury G, Morroy G, van Hoeven-Dekkers S, Botermans C, Veelenturf $C$, Bastiaens $M$, et al. An outbreak of scabies in multiple linked healthcare settings in the Netherlands. Infect Control Hosp Epidemiol. 2012;33:1047-50.

24. Tjioe M, Vissers WH. Scabies outbreaks in nursing homes for the elderly: recognition, treatment options and control of reinfestation. Drugs Aging. 2008;25:299-306.

25. Utsumi M, Makimoto K, Quroshi N, Ashida N. Types of infectious outbreaks and their impact in elderly care facilities: a review of the literature. Age Ageing. 2010;39:299-305.

26. Makigami K, Ohtaki N, Ishii N, Yasumura S. Risk factors of scabies in psychiatric and long-term care hospitals: a nationwide mail-in survey in Japan. J Dermatol. 2009;36:491-8.

27. Ariza L, Walter B, Worth C, Brockmann S, Weber ML, Feldmeier H. Investigation of a scabies outbreak in a kindergarten in Constance, Germany. Eur J Clin Microbiol Infect Dis. 2013;32:373-80.

28. Marotta M, Toni F, Dallolio L, Toni G, Leoni E. Management of a family outbreak of scabies with high risk of spread to other community and hospital facilities. Am J Infect Control. 2018;46:808-13.

29. Engelman D, Steer A. Control strategies for scabies. Trop Med Infect Dis. 2018;3:98.

30. Perciaccante A, Riva MA, Coralli A, Bianucci R. Scabies outbreak in the 14th century: clues from correspondence between poets. Am J Med. 2016;129:1136.

31. Micali G, Lacarrubba F, Verzi AE, Nasca MR. Low-cost equipment for diagnosis and management of endemic scabies outbreaks in underserved populations. Clin Infect Dis. 2015;60:327-9.

32. Salgado F, Elston DM. What's eating you? Scabies in the developing world. Cutis. 2017;100:287-89.

33. Sara J, Haji Y, Gebretsadik A. Scabies outbreak investigation and risk factors in East Badewacho district, Southern Ethiopia: unmatched case control study. Dermatol Res Pract. 2018;1-10.

34. Enbiale W, Ayalew A. Investigation of a scabies outbreak in droughtaffected areas in Ethiopia. Trop Med Infect Dis. 2018;3:114.

35. Romani L, Whitfeld MJ, Koroivueta J, Kama M, Wand H, Tikoduadua L, et al. Mass drug administration for scabies control in a population with endemic disease. N Engl J Med. 2015;373:2305-13.

36. Bouvresse $\mathrm{S}$, Chosidow O. Scabies in healthcare settings. Curr Opin Infect Dis. 2010;23:111-8.

37. Engelman D, Steer AC. Diagnosis, treatment, and control of scabies: can we do better? Lancet Infect Dis. 2018;18:822-3.

38. Hay RJ, Steer AC, Chosidow O, Currie BJ. Scabies: a suitable case for a global control initiative. Curr Opin Infect Dis. 2013;26:107-9.

39. Aussy A, Houivet E, Hébert V, Colas-Cailleux H, Laaengh N, Richard C, et al. Risk factors for treatment failure in scabies: a cohort study. $\mathrm{Br} \mathrm{J}$ Dermatol. 2019;180:888-93. 